\title{
A transição emocional materna no período puerperal associada aos transtornos psicológicos como a depressão pós-parto
}

\author{
The maternal emotional transition in the puerperal period associated with psychological \\ disorders such as postpartum depression
}

\section{La transición emocional materna en el período puerperal asociada con trastornos psicológicos como la depresión posparto}

Cynthia Araújo Frota ${ }^{1 *}$, Camila de Araújo Batista ${ }^{1}$, Rosa Irlania do Nascimento Pereira ${ }^{1}$, Ana Paula Costa Carvalho², Gabriel Lorran Freire Cavalcante ${ }^{3}$, Sudário Vítor de Aguiar Lima ${ }^{4}$, Caroline Natielle Rocha da Silva ${ }^{5}$, Luis Fernando Aguiar Araújo ${ }^{6}$, Fernando Antonio da Silva Santos ${ }^{7}$.

\section{RESUMO}

Objetivo: verificar evidências científicas sobre a ocorrência de transtornos psicológicos no período puerperal. Métodos: Realizou-se um estudo de revisão integrativa da literatura com busca de dados da Biblioteca Virtual e Saúde, LILACS e MEDLINE, tendo como descritores os termos Transtorno depressivo, Neurose Depressiva, Período pós-parto. Foram estabelecidos como critérios de inclusão: artigos científicos completos que contemplassem a temática e publicada em língua portuguesa, inglesa ou espanhola, no período de 2014 a 2018. Foram selecionados 21 estudos dos quais 95,2\% eram internacionais e publicados na Medline Resultados: Foi destaque nos estudos o grande risco que as mulheres apresentam para desenvolvimento de transtornos psicológicos no puerpério, que podem apresentar danos importantes a sua saúde sendo estes a ansiedade, psicose, transtorno bipolar, transtorno obsessivo-compulsivo, baixa autoestima e depressão pós-parto. Considerações finais: Destacou-se a necessidade de trabalhar estratégias para rastreio do risco de transtornos e melhor atenção à mulher, com cuidados integrais que atendam às suas necessidades gerais. Portanto, deve-se trabalhar ainda mais a temática, visto que no período puerperal muitas vezes não é oferecido um cuidado adequado.

Palavras-chave: Transtorno depressivo, Depressão pós-parto, Período pós-parto.

\begin{abstract}
Objective: This study aimed to verify scientific evidence on the occurrence of psychological disorders in the puerperal period. Methods: It`s an integrative review of the literature that was conducted with data search from the Virtual Library and Health, LILACS and MEDLINE databases, using the terms Depressive Disorder, Depressive Neurosis, Postpartum Period. Inclusion criteria were complete scientific articles that included the subject and published in Portuguese, English or Spanish during the period from 2014 to 2018. Twenty-one studies were selected, of which 95.2\% were international and published in Medline. Results: It was highlighted in the studies the great risk that women present for the development of psychological disorders in the puerperium, that it can present important damages to their health. such as anxiety, psychosis, bipolar disorder, obsessive-compulsive disorder, low self-esteem and postpartum depression. Final considerations: It was highlighted the need to work out strategies for the detection of risk of disorders and better attention to women, with comprehensive care that meets their general needs. Therefore, it is necessary to work even more on the theme, since in the puerperal period, adequate care is often not offered.
\end{abstract}

Key words: Depressive disorder, Depression postpartum, Postpartum period.

\footnotetext{
1 Faculdade Mauricio de Nassau (UNINASSAU), Teresina - PI. *E-mail: cynthiaaraujofrota@gmail.com

${ }^{2}$ Faculdade Estácio, Teresina - PI.

${ }^{3}$ Centro Universitário do Piauí (UNIFAPI), Teresina - PI.

${ }^{4}$ Centro Universitário Uninovafapi (UNINOVAFAPI), Teresina - PI.

${ }^{5}$ Faculdade de Educação São Francisco (FAESF), Pedreiras - MA.

${ }^{6}$ Faculdade Estácio, São Luís - MA.

${ }^{7}$ Centro Universitário de Ciências e Tecnologia do Maranhão (UNIFACEMA), Caxias - MA.
} 


\section{RESUMEN}

Objetivo: Verificar las evidencia científicas a cerca de la aparición de trastornos psicológicos en el período puerperal. Métodos: Se realizó un estudio integrador de revisión de literatura con búsqueda de datos de la Biblioteca Virtual y de Salud, LILACS y MEDLINE, utilizando los términos trastorno depresivo, neurosis depresiva, período posparto como descriptores.Los criterios de inclusión fueron: artículos científicos completos que cubrieron el tema y publicados en portugués, inglés o español, de 2014 a 2018.Se seleccionaron 21 estudios, el 95,2\% de los cuales fueron internacionales y se publicaron en Medline. Resultados: Se destacó en los estudios el gran riesgo que presentan las mujeres para el desarrollo de trastornos psicológicos en el puerperio, que pueden presentar daños importantes a su salud, como ansiedad, psicosis, trastorno bipolar, trastorno obsesivo compulsivo, baja autoestima y depresión posparto. Conclusión: Se destacó la necesidad de trabajar en estrategias para rastrear el riesgo de trastornos y una mejor atención para las mujeres, con una atención integral que satisfaga sus necesidades generales. Por lo tanto, el tema debe trabajarse más, ya que en el período puerperal, a menudo no se ofrece la atención adecuada.

Palabras clave: Trastorno depresivo, Depresión Posparto, Posparto.

\section{INTRODUÇÃO}

O período gestacional caracteriza-se como momento de grandes mudanças para a mulher tanto físicas, culturais e emocionais. Devido essas alterações é importante o acompanhamento da gestante com intuito de prever sérias complicações. Segundo estimativa da Organização Mundial da Saúde cerca de 500 mil mulheres no mundo morrem por ano por processos relacionado a gestação, destas $98 \%$ acontecem nos países em desenvolvimento (BOTELHO MN, et al., 2010).

As modificações do período gestacional e puerperal podem ser em nível social, fisiológico, familiar e psicológico. Para a mulher o período de gestação é visto como um momento de sonhos onde a mesma passa a imaginar, conhecer e tocar o filho. No entanto, em algumas situações este processo pode ser alterado. Após a gestação, em alguns casos é observado o desenvolvimento de sintomas logo após o nascimento do bebê. As transformações sofridas pela mulher no puerpério a deixam suscetível à maior frequência de agravos que são causas específicas de morbimortalidade materna (GIARETTA DG e FAGUNDEZ F, 2015; CORRÊA MSM, et al., 2017).

De acordo com Pina LNS e Loures MC (2014); no período puerperal ocorrem intensas mudanças, como alteração dos níveis de hormônios gonadais, nos níveis de ocitocina e no eixo hipotálamo-hipófise-adrenal. Diante de tantas alterações, é importante trabalhar a reorganização social e a adaptação da mulher a um novo papel, o que aumenta consideravelmente sua responsabilidade. Segundo Coker JL, et al. (2017); no período gestacional podem surgir alguns sinais e sintomas que podem ser característicos de transtornos psiquiátricos. Acredita-se que os mesmos podem ser de origem emocional e desenvolvendo sintomas depressivos. As intensas alterações que ocorrem podem repercutir no âmbito familiar, em alguns casos a gestante passa a sentir e imaginar sensações diferentes e assustadoras, como a sensação de desfiguração do corpo devido a gravidez. Dessa forma após o nascimento do bebê, pode acontecer que a mãe sinta o sentimento de solidão, vazio, momento esse em que as atenções estarão voltadas para o filho e não para ela, o que pode levar ao aparecimento de transtornos depressivos (TOLENTINO EC, et al., 2016).

De acordo com Pereira DJ (2017); o puerpério caracteriza-se por ser pontualmente após o parto e se estende até seis semanas, é nesse período que acontece à readaptação da mulher ao seu estado anterior à gravidez. Ele pode ser classificado em imediato, indo $1^{\circ}$ ao $10^{\circ}$ dia após o parto, e tardio, do $11^{\circ}$ ao $42^{\circ}$ dia. Este é um momento acompanhado por fortes mudanças sociais, físicas, hormonais e psíquicas, que reflete consideravelmente na saúde da mulher, tornando-a mais vulnerável nesse período a sofrer com a presença de transtornos mentais. Pesquisas mostram que cerca de 15 a $29 \%$ das puérperas sofrem algum transtorno psíquico, sendo a depressão puerperal o principal. De modo geral a depressão tem se tornado um problema comum na sociedade, com variação de 4 a $17 \%$ de ocorrência em mulheres no mundo todo. Em estudo de Azambuja CV, et al. (2017); verificou-se que, em um grupo de pessoas entre 20 e 69 anos, a prevalência dos sintomas depressivos foi notável, ansiedade $(57,6 \%)$, preferir ficar em casa $(54,3 \%)$, falta de disposição $(40,4 \%)$, falta de energia $(37,6 \%)$, pensar no passado $(33,8 \%)$, e tristeza $(29,4 \%)$, sintomas que podem 
manifestar-se na puérpera. Dessa forma, o transtorno depressivo, os sintomas da depressão podem manifestar-se no período gestacional e até quatro semanas após o parto.

Dessa forma os transtornos psicológicos, tais como a depressão são um grave problema para a saúde materna, com incidência em cerca de 10 a $20 \%$ das mulheres no pós-parto, com riscos para a saúde da mãe e para o desenvolvimento do bebê. Estimativas apontam que em 2020 a depressão será a segunda maior causa de morbidade no mundo. Em âmbito nacional, a prevalência dos sintomas está acima da média mundial, revelando dessa maneira a necessidade de atenção mais cautelosa a saúde materna por meio da atenção básica e outros serviços voltados para o cuidado das puérperas (PEREIRA DJ, 2017). Tendo como base as intensas transformações que ocorrem no parto e pós-parto, destaca-se a necessidade de acompanhamento da mulher por equipe de saúde multiprofissional preparada para atendê-la em suas necessidades biopsicossociais, uma vez que as mudanças ocorridas podem desencadear doenças no período puerperal, fazendo com que a mãe sinta-se incapaz de exercer o papel materno, sofrendo com variações de humor e sentimento de culpa, bem como risco de desenvolver sintomas psicóticos (PINA LNS e LOURES MC, 2014).

Dessa forma, este estudo justifica-se pela importância do período puerperal para a mãe e o risco que esta mulher apresenta para desenvolver problemas psicológicos graves, sendo necessário maiores discussões sobre a temática, que estimulem a busca de estratégias para enfrentamento desse problema. Portanto o estudo apresenta como objetivo verificar evidências científicas sobre a ocorrência de transtornos psicológicos no período puerperal.

\section{MÉTODOS}

Foi realizado um estudo de revisão integrativa da literatura, estabeleceu-se como questão norteadora do estudo: Quais as evidências científicas sobre a ocorrência de transtornos psicológicos no puerpério? A partir dessa questão foram identificados como descritores: Transtorno depressivo, Neurose Depressiva, Período pós-parto.

Figura 1 - Fluxograma representativo a respeito dos critérios de inclusão de exclusão.

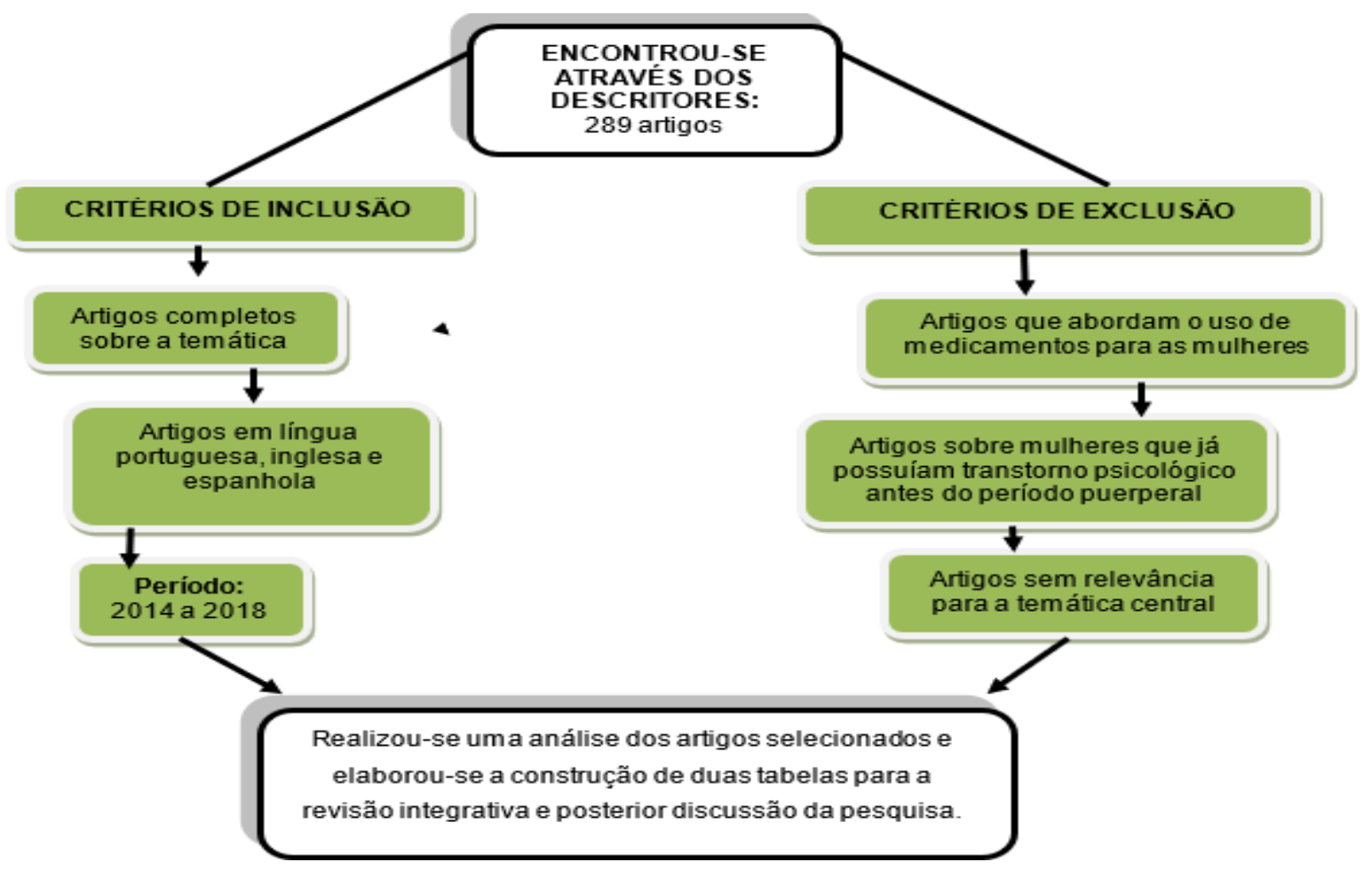

Fonte: Frota AC, et al., 2019. 
$\mathrm{Na}$ estratégia de busca utilizaram-se as seguintes formas booleanas como: OR e AND, para ajudar a encontrar os artigos da base de dados online. As bases de dados escolhidas foram a Biblioteca Virtual em Saúde com todos os colaboradores da rede Literatura Latino-Americana e do Caribe em Ciências da Saúde (LILACS) e Medical Literature Analysis and Retrieval System Online (MEDLINE).

\section{RESULTADOS}

Atendendo aos critérios foram utilizados 21 estudos com a finalidade de alcançar os objetivos propostos. Em seguida foram caracterizados em forma de quadros, de forma a traçar o perfil das publicações selecionadas.

O período gestacional é um momento bastante delicado com grandes mudanças, tanto no aspecto físico como psicológico, onde a mulher se encontra bastante emotiva, muitas mulheres tendem a não adaptar-se facilmente a esse novo momento de sua vida devido a todas as transformações e mudanças de sua rotina diária e assim tornando-as mais vulneráveis a desenvolverem distúrbios psicológicos durante a gestação como a depressão materna conforme descrito no Quadro 1.

Quadro 1 - Caracterização dos estudos de acordo com a sintomatologia, autores e resultados de cada estudo referente à gestação. Teresina, $\mathrm{PI}$.

\begin{tabular}{|c|c|c|}
\hline Sinais e sintomas & Autores & Resultados \\
\hline \multicolumn{3}{|c|}{$1^{\circ}$ trimestre } \\
\hline $\begin{array}{l}\text { Insatisfação com a aparência } \\
\text { corporal. }\end{array}$ & $\begin{array}{l}\text { SILVEIRA ML, et } \\
\text { al., (2015); }\end{array}$ & $\begin{array}{l}\text { Os achados foram consistentes em } \\
\text { diferentes aspectos da imagem corporal e } \\
\text { vários períodos de gravidez e pós-parto. }\end{array}$ \\
\hline \multicolumn{3}{|c|}{$2^{\circ}$ trimestre } \\
\hline $\begin{array}{l}\text { Comorbidade psiquiátrica, trauma } \\
\text { inicial, menor escolaridade, estado } \\
\text { civil, gravidez não planejada, } \\
\text { sintomas depressivos. }\end{array}$ & $\begin{array}{l}\text { COKER JL, et al., } \\
\qquad(2017) ;\end{array}$ & $\begin{array}{c}\text { As manifestações durante a gestação, de } \\
\text { problemas emocionais e sintomas } \\
\text { depressivos }\end{array}$ \\
\hline $\begin{array}{l}\text { História prévia de depressão, } \\
\text { transtorno de ansiedade comórbida, } \\
\text { histórias de violência doméstica. }\end{array}$ & $\begin{array}{l}\text { SHIVAKUMAR G, } \\
\text { et al., (2014); }\end{array}$ & $\begin{array}{l}\text { A progressão da depressão quando } \\
\text { tratadas com medicação antidepressiva } \\
\text { causa uma melhora dos sintomas na } \\
\text { gravidez e no pós-parto imediato. }\end{array}$ \\
\hline \multicolumn{3}{|c|}{ 3 trimestre } \\
\hline $\begin{array}{l}\text { Deficiência de vitamina D e B12; } \\
\text { sintomas depressivos. }\end{array}$ & $\begin{array}{l}\text { TRUJILLO J, et } \\
\text { al., (2018); }\end{array}$ & $\begin{array}{l}\text { Probabilidade aumentada de depressão } \\
\text { durante a gravidez e no período pós-parto. }\end{array}$ \\
\hline
\end{tabular}

Fonte: Frota AC, et al., 2019. 
Quadro 2 - Caracterização dos estudos de acordo com a sintomatologia, autores e resultados de cada estudo referente ao período puerperal. Teresina, PI. Pós-parto

\begin{tabular}{|c|c|c|}
\hline Sintomas hipomaníacos; episódios depressivos. & SHARMA V, et al., (2018); & $\begin{array}{l}\text { Mulheres com transtorno bipolar são frequentemente } \\
\text { diagnosticadas eroneamente }\end{array}$ \\
\hline $\begin{array}{l}\text { Perda de peso, tristeza, insônia, desorientação, perda } \\
\text { de memória, dificuldade de concentração. }\end{array}$ & $\begin{array}{l}\text { ACCORTT EE e WONG MS } \\
\qquad(2017) ;\end{array}$ & $\begin{array}{l}\text { Depressão e ansiedade são comuns durante a gestação e no } \\
\text { período puerperal. Os tratamentos não-farmacológicos para a } \\
\text { depressão, como terapia cognitivo-comportamental e tratamentos } \\
\text { complementares podem produzir alívio dos sintomas. }\end{array}$ \\
\hline Insatisfação com a aparência corporal. & $\begin{array}{l}\text { ROOMRUANGWONG C, et al., } \\
\qquad(2017) \text {; }\end{array}$ & $\begin{array}{l}\text { Está associada ao aumento dos escores de depressão e } \\
\text { ansiedade. }\end{array}$ \\
\hline $\begin{array}{c}\text { Perda perinatal prévia, eventos de vida estressantes e } \\
\text { falta de apoio social, transtornos depressivos } \\
\text { anteriores }\end{array}$ & VENKATESH KK, et al., (2017); & $\begin{array}{l}\text { Processo de busca dos sintomas da depressão durante os } \\
\text { períodos de anteparto e pós-parto é de real significância para a } \\
\text { sondagem da depressão pós-parto. }\end{array}$ \\
\hline $\begin{array}{l}\text { Ansiedade, preferir ficar em casa, indisposição, } \\
\text { cansaço, e tristeza. }\end{array}$ & AZAMBUJA CV, et al., (2016); & $\begin{array}{l}\text { Baixa autoestima, sentir-se inútil diante de cuidados básicos } \\
\text { corporais e alimentares, além da percepção da criança como } \\
\text { sendo "difícil". }\end{array}$ \\
\hline Estresse materno, episódios depressivos, baby blues. & OWORA AH, et al., (2016); & $\begin{array}{l}\text { Descoberta de casos de transtorno depressivo têm alto } \\
\text { desempenho no diagnóstico nos períodos periparto e pós-parto. }\end{array}$ \\
\hline $\begin{array}{l}\text { Declínio rápido do estrogênio, progesterona e cortisol } \\
\text { em combinação com estressores psicossociais. }\end{array}$ & BECKER M, et al., (2016); & $\begin{array}{l}\text { Depressão materna não tratada durante a gravidez e o período } \\
\text { pós-parto tem sido associada a resultados adversos tanto na } \\
\text { mãe quanto no bebê. }\end{array}$ \\
\hline $\begin{array}{l}\text { Presença de obsessões, compulsões, } \\
\text { Comportamentos repetitivos, perturbações. }\end{array}$ & $\begin{array}{l}\text { SHARMA V e SOMMERDYK C } \\
\qquad(2015) ;\end{array}$ & $\begin{array}{l}\text { O transtorno obsessivo-compulsivo (TOC) após o parto pode } \\
\text { ocorrer isoladamente ou em combinação com outros transtornos } \\
\text { psiquiátricos, como o transtorno depressivo. }\end{array}$ \\
\hline
\end{tabular}

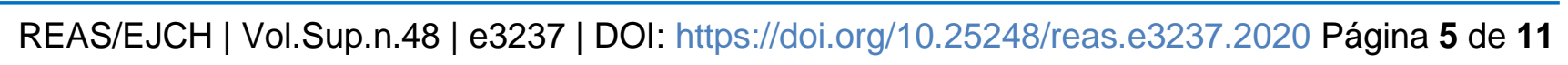




\section{Revista Eletrônica Acervo Saúde / Electronic Journal Collection Health | ISSN 2178-2091}

\begin{tabular}{|c|c|c|}
\hline \multicolumn{3}{|c|}{ Pós-parto } \\
\hline Medo, insegurança, desorganização, isolamento. & $\begin{array}{l}\text { GUTIÉRREZ-ZOTES A, et al., } \\
\qquad(2016) ;\end{array}$ & $\begin{array}{c}\text { Comportamentos como auto-responsabilização, auto-distração e } \\
\text { apoio emocional prejudicado foram agravadores dos sintomas } \\
\text { depressivos entre } 8 \text { e } 32 \text { semanas após o parto. }\end{array}$ \\
\hline $\begin{array}{l}\text { Má nutrição e ganho de peso inadequado; uso de } \\
\text { substâncias nocivas. }\end{array}$ & AWHONN (2015); & $\begin{array}{l}\text { A triagem sistemática na gravidez e no período pós-parto ajuda a } \\
\text { detectar sintomas precoces de sofrimento psiquiátrico perinatal. }\end{array}$ \\
\hline $\begin{array}{l}\text { Sentimento de culpa, comorbidade psiquiátrica, } \\
\text { neuroticismo, início precoce, cronicidade. }\end{array}$ & YANG F, et al., (2015); & $\begin{array}{l}\text { Depressão pós-parto em mulheres chinesas é tipicamente } \\
\text { crônica e grave, com fatores de riscos incluindo tensão pré- } \\
\text { menstrual grave e mais partos. }\end{array}$ \\
\hline Neuroticismo e ansiedade. & ILIADIS SI, et al., (2015); & $\begin{array}{l}\text { Mulheres não deprimidas que relataram altos níveis de } \\
\text { neuroticismo no final da gestação apresentavam alto risco de } \\
\text { desenvolver sintomas depressivos no pós-parto. }\end{array}$ \\
\hline $\begin{array}{l}\text { Tristeza, distúrbio de apetite e sono, fadiga, culpa, } \\
\text { dificuldades de concentração, ideação suicida. }\end{array}$ & HOERTEL N, et al., (2015); & $\begin{array}{l}\text { Sintomas depressivos em durante a gravidez e no período pós- } \\
\text { parto não difere daquele observado fora do período periparto. }\end{array}$ \\
\hline Transtorno do humor, depressão e transtorno bipolar. & KIMMEL M, et al., (2015); & $\begin{array}{l}\text { O uso de medicamentos psiquiátricos durante a gravidez pode } \\
\text { não proteger contra a depressão. }\end{array}$ \\
\hline $\begin{array}{c}\text { Ansiedade fóbica, ideação paranóide, humor } \\
\text { deprimido, diminuição do prazer e energia, agitação, } \\
\text { retardo psicomotor. }\end{array}$ & KETTUNEN P, et al., (2014); & $\begin{array}{l}\text { Depressão recorrente é comum. Todas as mães devem ser } \\
\text { rastreadas até a sexta semana pós-parto, o mais tardar. }\end{array}$ \\
\hline $\begin{array}{l}\text { Estresse psicossocial e depressão associada à } \\
\text { gravidez, comportamentos de risco à saúde, doença } \\
\text { médica e psiquiátrica pré-gravidez, doenças } \\
\text { relacionadas à gravidez e desfechos do nascimento. }\end{array}$ & KATON W, et al., (2014); & $\begin{array}{l}\text { Identificar fatores de risco sóciodemográficos e clínicos } \\
\text { específicos para a depressão pós-parto podem ajudar os } \\
\text { médicos a atingir a descoberta de casos de depressão em } \\
\text { mulheres grávidas. }\end{array}$ \\
\hline
\end{tabular}

Fonte: Frota AC, et al., 2019.

REAS/EJCH | Vol.Sup.n.48 | e3237 | DOI: https://doi.org/10.25248/reas.e3237.2020 Página 6 de 11 
Por meio da análise dos 21 estudos selecionados, foi organizado dois quadros (Quadro 1 e Quadro 2) relacionando os problemas aos transtornos psicológicos de mulheres de acordo com o período descrito no estudo. A grande maioria dos estudos concentrou sua atenção em destacar as repercussões dos transtornos psicológicos no período puerperal, o que se justifica por meio dos objetivos da pesquisa. Alguns poucos estudos relataram sintomatologias da gestação que contribuem para identificar o risco de transtornos psicológicos maternos no pós-parto. Os principais sinais e sintomas observados nos estudos foram: história familiar, picos de ansiedade, sofrimento, medo, desorientação, perda de peso, gravidez indesejada, história pregressa de depressão, distúrbio de apetite, distúrbio de sono, fadiga, culpa, dificuldades de concentração, ideação suicida, entre outros.

Após a análise do conteúdo dos estudos, as ideias e conclusões dos autores serviram para dar fundamento à discussão do tema com base nas evidências encontradas.

\section{DISCUSSÃO}

A partir dos quadros elaborados, a discussão baseou-se nos aspectos psiquiátricos no período gestacional e pós-parto, sendo dividida em duas categorias: (1) Sintomatologias depressivas no período gestacional e (2) Transtornos psicológicos no período puerperal.

A depressão durante a gravidez acarreta efeitos negativos, tanto de origens físicas como psicológicas, além de afetar no desenvolvimento da gestação. A gestante sente-se prescionada a lidar com a gravidez, não sabendo absorver as expectativas familiares e nem a buscar ajuda profissional na area da saúde (BORGES DA, et al., 2016).

A puérpera torna-se vulnerável ao desenvolvimento de depressão pós-parto e ansiedade, com as mudanças físicas, hormonais e psicologias. Evidenciado que se tornam maior o risco em mulheres de baixo nível socioecômico, mulheres com histórias anteriores de depressão e histórias anteriores de depressão pósparto (HARTMANN JM, et al., 2017). Tanto no pré-natal como no puérperio mostrou-se evidente a depressão e ansiedade, com fatores de riscos sendo baixo nível economico, problemas em gravidez anteriores e gravidez indesejada, mostrando a necessidade de olhar holistico sobre as gestantes durante o pré-natal (SILVA MMDJ, et al., 2015).

\section{Sintomatologias depressivas no período gestacional}

Os estudos abordaram a influência de sintomas gestacionais com repercussão no puerpério para a manifestação dos transtornos psiquiátricos. No primeiro trimestre, Silveira ML, et al. (2015); descreve em sua pesquisa que a imagem corporal da mulher, ao ser alterada como consequência da gestação e do parto, pode gerar insatisfação, que sua vez aumentando as chances de desenvolverem problemas psicológicos como a depressão materna.

Em estudo realizado com 842 mulheres, 22,3\% das puérperas relataram terem tido intenções suicidas, mesmo que $79 \%$ destas realizarem tratamento farmacológico para doenças psiquiátricas. O perfil destas mulheres que realizaram automutilação ou idealizaram o suicídio no pós-parto era de menor nível de educação, história de gravidez não planejada, bem como tentativas anteriores de suicídio, o que as torna mais suscetíveis a desenvolverem quadro de depressão, mostrando a influência que problemas e riscos presentes no período gestacional representam sinais para serem evidenciados no pós-parto, tornando-se fundamental identificar os fatores que podem predizer o aparecimento da depressão pós-parto (COKER JL, et al., 2017).

De acordo com Shivakumar G, et al. (2014); os principais fatores evidenciados no período gestacional, no segundo trimestre de gestação foram, a presença de sentimento de culpa das mulheres, morbidades psiquiátricas, neurotiquíssimo, maior intensidade de sintomas da tensão pré-menstrual, maior quantidade de partos, abuso sexual na infância e apoio social prejudicado.

A pesquisa de Trujillo J, et al. (2018); relacionou ainda a ocorrência de sintomas depressivos com aspectos nutricionais na gestação e no puerpério. Ao verificar dados sobre a influência de biomarcadores nutricionais, 
encontraram evidencias que a quantidade baixa de vitamina $D$ principalmente nas semanas finais da gestação pode estar ligado à depressão no pós-parto. Já no estudo de Venter M, et al. (2016) os autores observaram que não há valor de predição para o trauma infantil relacionado à depressão no pós-parto.

\section{Transtornos psicológicos no período puerperal}

Os transtornos psicológicos no puerpério que podem acometer as mulheres incluem problemas como a depressão pós-parto, o transtorno bipolar e psicose pós-parto, transtorno de ansiedade generalizada, transtorno de pânico, transtorno obsessivo-compulsivo, transtorno de ansiedade social, fobias específicas e transtorno de estresse pós-traumático (TEPT). Dados têm evidenciado que um percentual de 10 a $20 \%$ de mulheres apresentam problemas depressivos ou de ansiedade durante a gravidez ou no período pós-parto, tornando necessária a formulação de políticas de saúde e protocolos bem planejados para trabalhar a educação e triagem de mulheres nesse período de parto e puerpério (AWHONN, 2015).

Segundo Almeida NMDC e Arrais AR (2016); os transtornos de ansiedade também podem aparecer no pós-parto. Ele se revela devido um conglomerado de emoções, sentimentos oriundos da maternidade. Para o autor, o transtorno afetivo bipolar é uma doença onde a mulher pode apresentar intensa agitação psicomotora, ideação paranoide, variação de humor e transtornos depressivos. Ele salienta que existem fatores específicos do próprio indivíduo que podem proteger a mulher dos eventos depressivos, um desses fatores de proteção é resiliência, controle emocional, autonomia e expectativa de um futuro promissor.

O transtorno obsessivo-compulsivo (TOC) é um dos problemas que a mulher pode desenvolver após o parto, evidenciados por obsessões, compulsões, comportamentos repetitivos, perturbações e não raramente é confundido com o surgimento de transtorno depressivo. O surgimento de TOC nas puérperas descrito pelo autor são: medo de causar dano intencional ou acidental ao bebê, preocupação com o bebê morrer durante o sono, medo de ser julgada como mãe, limpeza, checagem, insegurança, e busca excessiva de segurança. O tratamento pode ser realizado em ambulatório, e nos casos de mulheres em risco de suicídio é recomendado tratamento hospitalar, bem como para aquelas com transtornos como depressão ou transtorno bipolar que são incapazes de realizar autocuidado adequado. $O$ tratamento medicamentoso e a psicoterapia poderão ser usados separados ou associados (SHARMA V e SOMMERDYK C, 2015).

A preocupação e insatisfação com a imagem corporal foi destacada por autores como sendo importante fator para aparecimento de transtornos no puerpério. No estudo de Roomruangwong C, et al. (2017) com 126 mulheres, 34,1\% apresentaram insatisfação com sua aparência corporal alterada pela gestação. Este fato pode estar associado a ocorrência de depressão e sintomas de ansiedade, sendo importante que a equipe médica esteja atenta a este sinal, uma vez que pode indicar o aparecimento de distúrbios do humor.

De acordo com Becker M, et al. (2016); a depressão pós-parto é a complicação mais comum após o parto, podendo manifestar-se em 10 a $15 \%$ da população feminina, a mudança que a maternidade traz consigo é um evento que pode ser estressante em razão das modificações de rotina e hábitos que acarreta. Ainda durante a gravidez, os sintomas depressivos são os principais sinais para a depressão pós-parto, além de ansiedade, história de doença psiquiátrica, fatores estressantes da vida, sinais de isolamento e baixa autoestima. Torna-se fundamental identificar os fatores que predizem o aparecimento da depressão pósparto. Segundo Yang F, et al. (2015); os principais fatores foram a presença de sentimento de culpa das mulheres, morbidades psiquiátricas, neuroticismo, maior intensidade de sintomas da tensão pré-menstrual, maior quantidade de partos, abuso sexual na infância e apoio social prejudicado. Iliadis SI, et al. (2015); destaca que o neuroticismo aumenta até quatro vezes o risco de sintomas depressivos no pós-parto em mulheres grávidas não deprimidas.

Já Hoertel N, et al. (2015) descreve ainda que a ocorrência de episódios depressivos no pós-parto de mulheres está frequentemente associada a sintomas obsessivo-compulsivos e psicóticos do que os episódios ocorridos antes do parto. Tais informações são necessárias para auxiliar os profissionais que trabalham no atendimento as gestantes e puérperas. Gutiérrez-Zotes A, et al. (2016); afirma que alguns aspectos tendem a diferenciar mulheres com e sem transtorno depressivo no pós-parto, fatores como a auto distração e o uso de substâncias, a autocensura, culpa e apoio emocional como fatores de risco para a depressão. 
Em estudo com mulheres avaliadas durante e após a gravidez, foi analisado o papel dos medicamentos no decorrer da depressão pós-parto. Verificou-se que, embora o uso de medicamentos psiquiátricos na gestação diminua o quadro geral de depressão, seu uso não garante proteção contra o aparecimento de depressão pós-parto em mulheres que tenham maior risco, como as que já apresentaram história de depressão pós-parto anterior (KIMMEL M, et al., 2015).

Em pesquisa de Katon W, et al. (2014); comparando mulheres sem sintomas depressivos no pós-parto e aquelas com depressão pós-parto, verificou-se que as que apresentavam os sintomas eram mais jovens, com maiores índices de desemprego, gestação associada a problemas depressivos, maior número de fumantes, utilizavam antidepressivos na gravidez e apresentavam mais morbidades na gestação, como diabetes e problemas neurológicos.

No estudo de Azambuja CV, et al. (2016); foi evidenciado que as mães que apresentaram depressão pósparto afirmam que seus filhos apresentam temperamento difícil. Contudo, foi observada também a situação inversa, onde o temperamento difícil da criança pode ser um fator desencadeante para a depressão pósparto.

Estudo realizado por Freitas MES, et al. (2016); a depressão pós-parto acomete cerca de 10 a 20\% das puérperas nos seis primeiros meses após o parto, os sinais e sintomas podem surgir nas primeiras quatro semanas após o parto e sendo mais evidente ao longo dos seis primeiros meses. Os sintomas variam desde violência doméstica, gravidez indesejada, disforia pré-menstrual, baixas condições socioeconômicas, bem como medo do bebê nascer com anormalidades. O diagnóstico da depressão no puerpério pode ser complicado pois alguns sintomas sempre são presentes no pós-parto como alterações do sono, apetite e fadiga.

A depressão puerperal pode levar graves problemas à saúde materno-fetal, Félix TA, et al. (2013) afirma que, no Brasil a depressão tem números alarmantes, sua incidência está de 10 a $42 \%$ com um nível alto e potencial para o desenvolvimento de problemas à saúde da mãe e do neonato, sendo importante observar os sintomas, sabendo que o diagnóstico precoce diminui as chances de agravo.

De acordo com Galvão ACC, et al. (2015); as gestantes podem demonstrar sinais de que é comum da depressão puerperal. Sentimento de culpa, ideação suicida, alterações do sono, desânimo, medo de machucar o bebê, diminuição da libido e do apetite, conflitos com o cônjuge, baixo apoio social, dificuldades econômicas, estresse e gravidez indesejada. Assim, é imprescindível que a equipe de saúde dobre a atenção as puérperas e seus bebês, sendo empática a sua situação, não cabendo aos mesmos julgamentos, mas, sim um olhar sensível e holístico.

Sánchez-Tapia FR, et al. (2017); em sua pesquisa com 321 mulheres que estavam com menos de um ano de pós-parto, verificou-se que $15,58 \%$ se suicidaram, 14,95\% acreditavam que era melhor morrer, $6,85 \%$ tiveram ideia suicida e $1,87 \%$ já haviam tentado suicídio. Verificou-se que as mulheres que praticaram suicídio eram separadas ou divorciadas. Em alguns casos os sintomas de depressão pós-parto e os sintomas típicos do puerpério são confundidos. São inúmeras as alterações que podem acontecer durante a gestação, tornamse imprescindível um criterioso acompanhamento psicológico antes do trabalho de parto. $\mathrm{O}$ acompanhamento pode corroborar para uma vivência menos conturbada, sendo possível prevenir eventuais alterações negativas do processo gravídico. Destaca-se a importância do apoio psicológico, sendo a enfermagem primordial em tal processo (ALMEIDA NMDC e ARRAIS AR, 2016; FREITAS MES, et al., 2016).

Evidências sobre a depressão pós-parto, destaca-se que não é um transtorno homogêneo e que apresenta variação em seus fatores de risco, sintomatologia, duração e gravidade. Assim, torna-se fundamental que as puérperas sejam rastreadas precocemente após o parto, bem como trabalhar a educação dessas mulheres quanto aos riscos e sintomas da depressão nesse período. Um dos problemas atuais nessa área é o diagnóstico errado atribuído às pacientes, que podem provocar consequências deletérias e até vezes fatais. A conscientização e capacitação dos profissionais de saúde sobre os transtornos psicológicos são fundamentais para melhorar 0 atendimento a essas pacientes e realização do psicoterapêutico e farmacológico de maneira adequada (KETTUNEN P, et al., 2014; SHARMA V, et al., 2018). 
É necessário que as equipes de saúde estabeleçam atendimento mais próximo das pacientes para prevenir problemas como transtornos de humor e ansiedade no puerpério com a utilização de ferramentas de triagem adequadas, educação sobre saúde mental materna. Destaca-se que é essencial a realização de triagem no pós-parto para identificar mulheres com maiores riscos de apresentação do transtorno (ACCORTT EE e WONG MS, 2017; OWORA AH, et al., 2016; VENKATESH KK, et al., 2017).

\section{CONSIDERAÇÕES FINAIS}

Observou-se que o puerpério se caracteriza por episódios de instabilidades emocionais e vulnerabilidade da mulher, relacionadas às mudanças e as novas adaptações que acontecem no âmbito familiar e psicológico. Constatou-se no estudo a depressão pós-parto, sendo o transtorno mais abordado, podendo causar graves repercussões à saúde da mulher. Conclui-se também que os profissionais de saúde têm um papel primordial no cuidado às mulheres no período gravídico, parto e pós-parto, torna-se fundamental que a equipe de saúde vise ações que proporcione educação continuada É de suma importância que a temática seja mais trabalhada, pois no puerpério muitas vezes não é oferecido um cuidado adequado. O profissional de saúde deve ter um olhar holístico e empático, a fim de ajudar a mulher a passar pela fase de novas experiências e descobertas.

\section{REFERÊNCIAS}

1. ACCORTT EE, WONG MS. It is time for routine screening for perinatal mood and anxiety disorders in obstetrics and gynecology settings. Obstetrical \& gynecological survey, 2017; 72(9): 553-568.

2. ALMEIDA NMDC, ARRAIS AR. O pré-natal psicológico como programa de prevenção à depressão pósparto. Psicologia: Ciência e Profissão, 2016, 36(4): 847-863.

3. AWHONN - Association of Women's Health, Obstetric and Neonatal Nurses. Mood and Anxiety Disorders in Pregnant and Postpartum Women. Journal of Obstetric, Gynecologic \& Neonatal Nursing, 2015; 44(5): 687-9.

4. AZAMBUJA CV, et al. Depressão pós-parto materna e bebês com malformações: revisão sistemática. Aletheia, 2016; 49(2): 30-7.

5. BECKER M, et al. Depression During Pregnancy and Postpartum. Curr. Psychiatry Rep, 2016; 18(3): 32.

6. BORGES DA, et al., A depressão na gestação: uma revisão bibliográfica. Revista de iniciação científica da libertas, 2016; 1(1).

7. BOTELHO NM, et al. Aspectos clínico-epidemiológicos das mulheres pós-abortamento em hospital de referência. Rev para med, 2010; 24(1).

8. COKER JL, et al. Rating scale item assessment of self-harm in postpartum women: a cross-sectional analysis. Archives of women's mental health, 2017; 20(5): 687-694.

9. CORRÊA MSM, et al. Acolhimento no cuidado à saúde da mulher no puerpério. Cadernos de Saúde Pública, 2017; 33(3): e00136215.

10. FÉLIX TA, et al. Atuação da enfermagem frente à depressão pós-parto nas consultas de puericultura. Enferm. glob., Murcia, 2013; 12(29): 404-419.

11. FREITAS MES, et al. Análise dos fatores de risco associados à depressão pós-parto: revisão integrativa. Revista de Atenção à Saúde, 2016; 14(48): 94-98.

12. GALVÃO ACC, et al. Prevalência de depressão pós-parto e fatores associados: revisão integrativa. Revista Ciência\&Saberes-Facema, 2015; 1(1): 54-58.

13. GIARETTA DG, FAGUNDEZ F. Aspectos psicológicos do puerpério: Uma revisão. Psicologia, 2015; 1(1): 1-8.

14. GUTIÉRREZ-ZOTES A, et al. Coping strategies for postpartum depression: a multi-centric study of 1626 women. Archives of women's mental health, 2016; 19(3): 455-461.

15. HARTMANN JM, et al. Depressão entre puérperas: prevalência e fatores associados. Cadernos de Saúde Pública, 2017; 33: e00094016.

16. HOERTEL N, et al. Are symptom features of depression during pregnancy, the postpartum period and outside the peripartum period distinct? Results from a nationally representative sample using item response theory (IRT). Depression and anxiety, 2015; 32(2): 129-140.

17. ILIADIS SI, et al. Personality and risk for postpartum depressive symptoms. Archives of women's mental health, 2015; 18(3): 539-546

18. KATON W, et al. Predictors of postpartum depression. Journal of women's health, 2014; 23(9): 753-759.

19. KETTUNEN $P$, et al. Is postpartum depression a homogenous disorder: time of onset, severity, symptoms and hopelessness in relation to the course of depression. BMC pregnancy and childbirth, 2014; 14(1): 402.

20. KIMMEL M, et al. Family history, not lack of medication use, is associated with the development of postpartum depression in a high-risk sample. Archives of women's mental health, 2015; 18(1): 113-121.

21. OWORA AH, et al. Summary diagnostic validity of commonly used maternal major depression disorder case finding instruments in the United States: a meta-analysis. Journal of affective disorders, 2016; 205: 335-343. 
22. PEREIRA DJ. Depressão puerperal: fatores de risco e ações do enfermeiro no diagnóstico precoce. 2017. Monografia - Centro Universitário São Lucas, Porto Velho. 2017.

23. PINA LNS, LOURES MC. Puérpera com Depressão Pós-Parto: a influência na relação com o bebê. Revista EVSRevista de Ciências Ambientais e Saúde, 2014; v. 41, n. 2, p. 341-357.

24. ROOMRUANGWONG C, et al. High incidence of body image dissatisfaction in pregnancy and the postnatal period: Associations with depression, anxiety, body mass index and weight gain during pregnancy. Sexual \& Reproductive Healthcare, 2017; 13: 103-109.

25. SÁNCHEZ-TAPIA FR, et al. Frecuencia de tendencias suicidas (suicidalidad) y factoresasociadosenmujeres durante el primer añoposparto. Revista de Neuro-Psiquiatría, 2017; 80(3): 172-180.

26. SHARMA V, et al. Should all women with postpartum depression be screened for bipolar disorder?. Medical hypotheses, 2018; 118: 26-28.

27. SHARMA V, SOMMERDYK C. Obsessive-compulsive disorder in the postpartum period: diagnosis, differential diagnosis and management. Women's Health, 2015; 11(4): 543-552.

28. SHIVAKUMAR G, et al. Progression of major depression during pregnancy and postpartum: a preliminary study. The Journal of Maternal-Fetal \& Neonatal Medicine, 2014; 27(6): 571-576.

29. SILVEIRA ML, et al. The role of body image in prenatal and postpartum depression: a critical review of the literature. Archives of women's mental health, 2015; 18(3): 409-421.

30. SILVA MMDJ, et al. Ansiedade e depressão na gravidez: caracterização de gestantes que realizaram pré-natal em unidades públicas de saúde. Journal of Nursing UFPE/Revista de Enfermagem UFPE, 2015.

31. TOLENTINO EC, et al. Depressão pós-parto: conhecimento sobre os sinais e sintomas em puérperas. Rev. Ciênc. Saúde Nova Esperança, 2016; 14(1): 59-66.

32. TRUJILLO J, et al. A systematic review of the associations between maternal nutritional biomarkers and depression and/or anxiety during pregnancy and postpartum. Journal of affective disorders, 2018; 232: 185-203.

33. VENKATESH KK, et al. Improving discrimination in antepartum depression screening using the Edinburgh Postnatal Depression Scale. Journal of affective disorders, 2017; 214: 1-7.

34. VENTER M, et al. Impact of childhood trauma on postpartum depression: a prospective study. Archives of women's mental health, 2016; 19(2): 337-342.

35. YANG F, et al. Clinical features of and risk factors for major depression with history of postpartum episodes in Han Chinese women: a retrospective study. Journal of affective disorders, 2015; 183: 339-346. 